V.

\title{
A Case of Gangrene of the Leg from Thrombosis During Pregnancy.
}

\author{
By Rorert J ARDine, M.D., etc. \\ Senior Physician to the Glasgow Maternity Hospital.
}

Mrs. McQ., primigravida, æt. 22, within a fortnight of full time, was sent to the Maternity Hospital, by Dr. Roxburgh of Troon, on June 11th, 1907.

Patient's History. The previous health has been excellent, as the patient cannot remember ever having had any serious illness. She has never had rheumatism, and has never suffered from breathlessness or palpitation to indicate that there was any cardiac lesion. She was married about a year ago, and is now within a fortnight of the date on which she expects to be confined. Up to the last month her health had been excellent; then she began to suffer from cramps in her right leg and foot, alternating with sensations of numbness and tingling. These sensations were localized in the foot and lower two-thirds of the leg. Up to the date of admission there had never been any pain in the thigh.

On June 5th, the sensations and discomfort of which she had hitherto taken very little notice were succeeded by severe pain in the toes and foot. The pain was agonizing, and came on in paroxysms lasting for some minutes at first, and later for somewhat longer periods. She was seen by Dr. Roxburgh and his assistant. She was kept in bed and given morphia hypodermically to ease the pain, and fomentations were applied to the leg.

The patient was admitted to the Hospital six days after the severe pain began. An examination of the right foot and leg revealed that they were white, bloodless, and icy cold. Tactile sensation was quite gone in the foot and for some 4 inches above the ankle. No pulsation could be felt in the posterior tibial or dorsalis pedis arteries, but it could be faintly felt in the popliteal artery and distinctly in the femoral artery in Scarpa's triangle.

The pulse was 98 per minute and the temperature $98^{\circ} \mathrm{F}$. The heart sounds were slightly roughened in the aortic area, but otherwise pure. The lungs were normal. There was a slight trace of albumen in the urine.

The limb was carefully cleansed and rendered as aseptic as possible, and then enveloped in iodoform gauze and two layers of Gamgee tissue.

June 12th. The patient had several severe attacks of pain during 
the night, and two hypodermic injections of $\frac{1}{4}$ grain of morphia were given, but she obtained very little sleep. Several patches of purplish discolouration were beginning to show on the leg. A vaginal examination revealed that the os would admit one finger. The head presented. The fretal beart was audible.

June 13th. Early this morning labour commenced, and by 9 a.m. the os was fully dilated. As the patient was very weak delivery was effected by forceps. The child was hydrocephalic and did not survive more than a fow minutes. The perineum was slightly torn and it was stitched.

June 15th. Pulsation has now ceased in the femoral artery, and the whole of the thigh is cold to the touch. Discolouration has become apparent above the knee.

June 16th. Pulsation is still absent in the femoral artery, but the thigh is quite warm, and the discolouration has not spread any further. Sensation is completely lost in the lower two-thirds of the leg, but above that, and in the thigh, it is perfect. A distinct systolic murmur can be heard at the base of the heart to-day.

June 23rd. The patient has been fairly well. For a few days the temperature rose to about $100^{\circ} \mathrm{F}$., and the lochia were slightly foetid. An intra-uterine douche was given, and the temperature soon came down to normal. The pulse rate has varied between 110 and 120. On June 20th the dressing on the leg was changed. Large bullæ have formed on the leg, and the part in contact with the bed is sedematous and beginning to break. The thigh is quite warm, and sensation is perfect above the head of the tibia.

July 2nd. The patient has improved in strength during the last fow days. The line of demarcation is showing just above the knee. To-day she was transferred to the Royal Infirmary to Mr. Hogarth Pringle's wards.

Mr. Pringle has kindly furnished me with notes of the case, while the patient was under his care.

July 5 th. The right lower limb was amputated just below the trochanter by the circular method, as this gave the best covering for the stump, and at the same time allowed the margin of the wound to be as little removed from the source of its vascular supply as possible. All the vessels in the face of the stump were thrombosed, and only the femoral artery and vein were tied. No tourniquet was used, but the abdominal aorta was compressed during the operation.

On July 9th the patient complained of severe pain in the left foot and leg, and pulsation in the tibial arteries at the ankle could not be felt. The limb was covered with cotton wool, and on July 11th pulsation could not be felt in the femoral artery in Scarpa's triangle. The pains in the leg were severe and the foot was cold. On July 13th the femoral pulse could be recognized again. The wound was dressed, and it was seen that the flap was becoming gangrenous (dry) 
here and there. The gangrene spread so far as to destrciy the covering of the stump, and the patient, who refused to have any further operation to remedy this, left the hospital on August 29th. The circulation in the left limb had recovered, and the foot become warm, but up to the time the patient left the hospital the pulse in the tibial arteries was not certainly recognized.

I am quite at a loss to account for the thrombosis which occurred in this case. The cardiac condition could not have caused it, as the lesion was so slight that at times no murmur could be heard. The process of blocking seems to have been a gradual one of some weeks' duration. The pain and discomfort only became very marked a few days before the patient was sent to the Maternity Hospitul. By that time the tibial arteries were blocked, and there was only faint pulsation in the popliteal. Complete blocking of the fernoral artery quickly followed.

The amputation was done without the application of a tourniquet, and $I$ am quite sure that the compression of the aorta might also have been dispensed with. Mr. Pringle dissected the artery out of the leg after removal as far down as the bifurcation into the tibials. It was completely filled with clot.

The patient had a very narrow escape from losing hir other leg from the same cause, but fortunately the circulation was re-established in time to prevent gangrene. It was unfortunate that she would not allow Mr. Pringle to make new flaps and get the end of the stump properly covered.

I do not think that the hydrocephalic condition of the child had any bearing on the condition. 